\title{
Oral health promotion: expanding the boundaries of knowledge*
}

\author{
Lilian Marly de Paula \\ Nilce Santos de Melo \\ Heliana Dantas Mestrinho \\ (organizers)
}

Univ de Brasília, Brasília,

DF, Brazil.
* Summary of the discussions held at the "Oral Health Promotion: Expanding the Boundaries of Knowledge" International Symposium, organized by the Brazilian Association for Oral Health Promotion (ABOPREV), June 30 to July 2, 2011, Brasília, DF, Brazil.

Corresponding Author: Lilian Marly de Paula

E-mail: lilianmpaula@gmail.com

Submitted: Dec 12, 2011

Accepted for publication: Jan 02, 2012 Last revision: Aug 20, 2012
$\mathbf{T}$ he following professors participated in the symposium discussions as panelists: Maria Fidela de Lima Navarro (Univ de São Paulo, Bauru, SP, Brazil); Joana Carvalho (Univ de Louvain, Louvain, Belgium); Alexandre Rezende Vieira (Univ of Pittsburgh, Pittsburgh, PA, USA); Mário Taba Júnior (Univ de São Paulo, Ribeirão Preto, SP, Brazil); Tiago Sousa Coelho (Univ de Brasília, Brasília, DF, Brazil); and the following professors participated as moderators: Jaime Aparecido Cury (Univ de Campinas, Piracicaba, SP, Brazil); Nilce Tomita (Univ de São Paulo, Bauru, SP, Brazil); Lilian Marly de Paula (Univ de Brasília, Brasília, DF, Brazil).

Jaime Cury: The analysis of the "SB Brazil 2010" National Oral Health Survey shows a steady reduction in the number of caries in our country. Nevertheless, there are still regional disparities, especially when we consider the Northern and Northeastern regions of the country, where the indicators are worse. What solutions, whether genetic, behavioral or political in nature, do the panelists propose to reduce this imbalance?

Alexandre Vieira: The disparity problem is mainly related to the unequal allocation of resources and access to services, among other things. Unfortunately, the underlying genetic component does not provide immediate answers to this issue. My studies analyzing individual susceptibility as a risk factor for dental caries are still at an early stage and cannot yet be translated into clinical practice.

Joana Carvalho: In my opinion, we have to work together with other health areas, as well as with the families, in order to reduce dental caries and to promote better healthcare in these regions. I think this is the simplest way of working with primary care, i.e., through education and basic oral healthcare measures. These simple actions have proven very effective in promoting long-term qualitative and quantitative improvements in this area. I believe the best thing we can do, practically speaking, is to educate people about the importance of tooth brushing and the use of toothpaste. This would be my recommendation.

Maria Fidela: Social determinants are complex. There is no single factor that can determine how people will react. If the population lacks basic understanding about this matter, even the simplest educational actions may be hard to assimilate. It's a known fact that the low-income populations are more vulnerable to a larger number of diseases, including oral diseases. Oral health cannot be analyzed as an isolated element. It is a part of a person's overall health condition. In order to change this complex situation, we need sound public policies. In that sense, our country has created the Prouni (University for All Program) and the Bolsa Familia cash transfer program. These initiatives are enabling changes to be 
made in the current scenario. The good economic performance of the country is another aspect to be considered.

The population has also benefited from the creation of new job positions. This has also had an impact on employee wages. This is one of the most important factors to guarantee a person's well being. I'm particularly concerned about the situation in the North and Northeast, considering that these regions have reported the worst indicators in the country. This is especially true for the North, where we have been seeing little improvement in the indexes, when compared to other regions. This is partly due to the fact that other regions have better access to the necessary resources and have implemented other measures as well.

The Brasil Sorridente (Smiling Brazil) Program addresses the fluoridation issue. How are we going to provide fluoridation to riverside populations, for instance? The delivery of the dental kit, which contains a toothbrush, toothpaste, and dental floss, has already had a positive impact on the oral health indicators. The distance-learning program is also an important initiative for a country as large as Brazil, because it creates opportunities for those who live in remote areas.

Currently, we have been facing a situation in which professors teaching in Dental Schools do not accept the fact that it is no longer necessary to teach so many specialized procedures. We had problems implementing the Programa Saúde da Família (Family Health Program) simply because the practitioners were not prepared to work with primary care.

In some cases, these professionals were unable to perform simple dental restorations because they were not trained to work in the existing facilities and conditions. This does not mean, however, that we must reduce the quality of the services being rendered. The idea here is that we must work as a part of a larger and more integrated health team, which must be prepared to identify problems and find suitable solutions.

In sum, I believe that there is a great deal that can and should be done in the area of dentistry. We must be aware of our own reality in order to improve the existing services, and also must innovate.
We can provide high-quality services, such as firstrate restorations, in adequate conditions, by using ART, etc. Finally, we must also be patient, because people tend to resist change. We believe we are on the right track and we are providing good services. Furthermore, the situation tends to improve, and we must learn to be patient with some of our colleagues who do not share the same ideas about the way forward in our country.

Tiago Sousa: An academic, professional and industrial change in perspective must occur before we can actually change the current situation in Brazil. This became quite clear to me during President Lula's administration, when a decision was made to decentralize the governmental initiatives, focusing on the Northeast. This is something that we still lack in the Central-West and Northern regions of the country: changes driven by social and political actions.

Regarding the behavioral issues, I believe we will achieve our goals if we are able to understand the social and cultural aspects of each region. We must talk to the hands-on practitioners to check if the policies were successfully implemented. With this in mind, we must design dental health policies using a bottom-up approach, and not the other way around.

Mário Taba: In my opinion, we must focus our resources in the regions that face the largest challenges, in an effort to mitigate these disparities and reduce the incidence of such problems.

Lilian de Paula: Unfortunately, dental practitioners are trained to work apart from other health professionals, and, consequently, we are in the habit of solving dental health problems on our own. This is a big mistake. As was already mentioned, we must work together with other professionals; otherwise oral health will not survive in the area of health promotion. To do this, we must first acknowledge that we do not perform an isolated activity. Secondly, we must enhance health promotion. This is a challenge, especially because of the dental practitioner educational model we have in place, which stresses the need for specialized training. That makes it very difficult for students to understand their reality. When they actually start working, they will come to realize that health promotion solves the problem. 
In spite of this, they are trained to adopt a specialist approach and focus on the techniques. This makes it hard for them to take a step back and simplify the process. In turn, this discourages them from pursuing a career in the area.

Jaime Cury: Analysis of the "SB Brazil 2010" revealed there was no improvement concerning gingivitis and periodontal diseases. Considering that gingivitis is a direct consequence of poor oral hygiene and dental care, how would you explain this paradox?

Mário Taba: I believe this paradox results from the fact that the tooth is inside the oral cavity and that it is exposed to etiologic agents. What worries me about this scenario is perhaps our lack of preparation to face the avalanche of new problems that will arise from this situation. It also shows that, although some caries-prevention actions are being very effective, periodontal diseases may require special attention. We must take this new trend into consideration.

Maria Fidela: In the university outpatient clinics, we often observe that they emphasize the importance of tooth brushing, but they don't stress the need to use dental floss. In my opinion, dental floss and alternative flossing methods are a fundamental part of oral hygiene. Therefore, flossing should be stressed as an important measure in dental care.

Mário Taba: Certainly, dental floss plays an extremely important role in dental hygiene. I wonder what the real impact of dental flossing is on gingivitis, in terms of large populations. Of course, this will help in areas with more retention of biofilm, but does it have this coverage, and actually have an effect on gingivitis?

Jaime Cury: The issue being discussed here is that we had a large reduction in dental caries, but this was not followed by a corresponding reduction in gingivitis among young people. So how would you explain the fact that a reduction in caries did not result in an improvement in gingival health? With the purpose of providing additional information to support the debates, it's important to highlight that even in the most developed countries, only ten percent of the population uses dental floss.

Joana Carvalho: European epidemiological studies show that periodontal disease prevalence is not high, in spite of the fact that only $18 \%$ of the population uses dental floss. Therefore, I believe flossing plays a relevant role concerning gingivitis, but is not as relevant in terms of periodontitis.

Tiago Sousa: In his presentation, Prof. Roncali said that the DMFT index indicates that the missing component has decreased considerably. This means that we've been able to keep more teeth in the oral cavity, and, consequently, we're more susceptible to gingivitis.

Jaime Cury: We all brush our teeth, but how many of us actually clean our teeth perfectly? We recently published a paper showing the effect of fluoride toothpaste. It reveals that people do not brush their teeth thoroughly; consequently, some residual biofilm is left after brushing. Fluoride penetrates the biofilm and this has an effect on the reduction of caries. Apart from that, there's also a slight reduction in plaque, because fluoride will compensate for the effects of sugar. Therefore, when discussing gingivitis, we must stress the importance of improving the quality of dental hygiene, and this involves both periodontal diseases and caries.

Audience: Dr. Mário Taba, regarding the genetic determination of periodontitis, which was well illustrated in the case of the three sisters, isn't the transmissibility of periodontal pathogenic microorganisms in the intra-family environment an issue to be considered?

Mário Taba: Certainly, there is transmission of periodontal pathogens among family members. This hypothesis is supported by several studies. The presence of the bacterium itself is not a factor that will cause the problem. A strong genetic component is observed when this problem occurs among members with a similar genetic background.

Audience: Prof. Joana and Prof. Mário, many practitioners choose to examine patients in two consecutive sessions, first to identify the existence of caries or periodontal diseases, and, subsequently, to follow up on the progression of these conditions. Do you consider this a good practice?

Mário Taba: I do. Nevertheless, I believe that by using the appropriate tools, we can monitor the biological event through biological indicators. We need 
to find the ideal marker to answer that question. We can't wait for the disease to progress in order to start treatment. This is something needed in all areas.

Joana Carvalho: I agree. Regarding dental caries, there are clinical characteristics that we can use to identify the activity of a lesion. For example, a white, rough and opaque lesion is considered active. If, on the other hand, we see the opposite situation, the lesion is inactive. At first, we can determine the disease activity. However, there are intermediary stage lesions. In these cases, we must examine the patient two or three times to check if this lesion is developing or not. At the inter-proximal level, it is impossible to really see the activity using only an $\mathrm{x}$ ray at a given time. In most cases, more than one appointment will be necessary in order to determine the activity of a lesion.

Audience: Prof. Joana, do you think epidemiological research without calibration is reliable?

Joana Carvalho: It would be better to have calibration; however, this is not feasible for large epidemiological studies. In order to bypass this situation, we must train the professionals and make them understand the concepts and criteria. Each person will apply these concepts in the best way possible. Will we lose accuracy in the results? Yes, they will be less accurate. Now, I'm not quite sure if this lack of accuracy is really that relevant; however, when we are dealing with scientific studies we have to do the calibration.

Nilce Tomita: Just to add to what was said, I think we should mention the Brazilian experience in regard to the "SB Brazil" survey carried out from 2000 to 2003, as well as "SB Brazil 2010". In this case, we had enough time to prepare and train the professionals to work with the same tools, using standardized criteria and codes proposed by the World Health Organization. After that, we had a skill-building course for the calibration trainers, who were responsible for disseminating this information and providing guidance to the on-site examiners.

Joana Carvalho: The clinical examinations for epidemiological surveys should be a subject taught in the cariology course. I think that the quality of the surveys will be better once these practices are implemented at the university level. And that's what we're trying to do in Belgium.

Tiago Sousa: We're rethinking our syllabus and we have already implemented it. The way we see it, the issue of calibration in epidemiological surveys of large populations is important too. Sure, the financial investment will be much greater, but I think it is a feasible effort.

Audience: Dr. Joana, in the epidemiological study being conducted in Belgium, are you carrying out biofilm assessment? Could you discuss the challenges of performing biofilm assessment for a national epidemiological study, and the importance of incorporating this assessment into a national survey of oral health? Could you discuss the caries index being used? Could you discuss the need for cleaning prior to the dental caries examination? What are the problems of not cleaning prior to a dental examination?

Joana Carvalho: We decided to hold a survey including biofilm as a measure of oral hygiene, because we want to determine how thoroughly the population brushes its teeth. The difficulty is the same as that of any other measure. Regarding the need for cleaning prior to a dental examination, this is related to the level of the disease we want to detect. For example, in our study we decided to detect caries only on the cavity level; for this reason, cleaning prior to the examination was not mandatory.

Audience: Dr. Alexandre, for radiation caries, can we apply your aquaporin model? Have you used in your research streptococci mutans as an indicator of cariogenic activity? Could you discuss what the problems are in relation to using this indicator?

Alexandre Vieira: The issue of aquaporin with radiation caries has already been studied. Radiation therapy, used to treat cancer, causes the salivary gland to shrink and stop working. We proposed reversing this process by introducing aquaporin in a salivary gland that was injured or damaged due to radiation.

As for the question about streptococci mutans, we do not use it as an indicator of the disease, although it is an important component in triggering the disease. 
Joana Carvalho: Just to add to what Alexandre just said, we have to remember that this specific group of bacteria is associated with the incidence of caries, but it's not the only group involved. On the other hand, it's important to identify patients with large numbers of streptococci mutans, because this is associated with the development of new carious lesions.

Audience: Dr. Maria Fidela, the "SB 2010" data revealed that people have more caries in the North and Northeast, when compared to the statistics from the South, Southeast and Center-West. Would it be of interest to use ART to solve the problem of restorations in these regions?

Maria Fidela: I just wanted to point out that, when dealing with any kind of disease, one important aspect is that people must be aware of what causes the disease. For instance, when we stress the importance of adequate oral hygiene before going to sleep at night, people are usually very surprised. They are not aware of the fact that they have to be more thorough in tooth brushing at night because of a reduction in the flow of saliva when we sleep.

On the issue of disparity, we know that the DMFT index presents a historical overview of the caries situation in the country. Therefore, the index will not decrease. It can either stop progressing or it can increase. It will not drop. It reflects the situation regarding caries, missing teeth or fillings, and that's something we must take into consideration.

Something that we can do is to improve practitioner training in each region, so that these professionals can provide better dental care to patients. Regarding ART, it is a technique that was thought to have a limited lifespan in the beginning, and now it's being acknowledged as a more permanent or definitive treatment. The literature still lacks conclusive findings about the long-term use of ART. I think professional training is vital for the success of ART.

Joana Carvalho: I'd like to comment on the issue that Fidela spoke about, regarding the fact that the DMFT and DMFS indexes do not decrease. I agree with the statement, as long as we are considering caries. However, when we work with records of non-carious lesions, we can actually have a decrease in the index. A lesion may regress; it may decrease; it may even disappear. My intervention is just to add to the previous comment.

Jaime Cury: The issue of thorough tooth brushing at night, brought up by Dr. Fidela, is extremely important. A report presented in the ORCA congress, in Lithuania, mentioned a study revealing that if a person had only two opportunities to brush their teeth, or only one opportunity to brush their teeth per day, which would be the best moment to do so? The results of the study demonstrated that the benefits obtained from night brushing with fluoride toothpaste are superior both in terms of enamel and dentin when compared to morning brushing.

Joana Carvalho: I'd just like to inform you that when we conduct epidemiological studies and we ask patients how many times a day and for how long they brush their teeth, most people who say they brush their teeth once a day will report doing so in the morning. And this is something that happens quite frequently with teenagers and young adults, because most people brush only once a day. When we say that in Brazil the recommendation is to brush three times a day, people are surprised, because this is not something feasible in other countries, such as European countries. Tooth brushing is not practiced in schools, for instance, and, consequently, people brush twice a day.

Jaime Cury: Actually, nobody recommends that people brush their teeth three times a day. The population is in the habit of brushing their teeth to remove food fragments from their mouth and to ensure good breath, and this practice is usually associated to the three main meals we have.

Audience: With the evolution of knowledge in the genetic field and especially because of the issue of personalized medicine, is it possible that we may replace forms and surveys in the future with genetic readings?

Alexandre Vieira: Yes. There is a philosophical trend to move in that direction. What we envisage for the future is that we are going to have a chip, an instrument that will measure a number of factors for the most common diseases that cause the greatest amount of "burden," i.e., the greatest amount of pressure on the healthcare system. Whether this 
means we will eliminate certain records that we normally use, such as paper forms and questionnaires about a patient's medical history, is something we are still assessing. The answer is both yes and no. Maybe in the future we will rely entirely on electronic and digital records, but I believe we will still have to reach conclusions based on our one-to-one contact with the patients. Nonetheless, the decisions will be largely based on the presence of certain genetic susceptibilities. This applies, for example, to cancer therapy and the response to medication, to therapy for other diseases, and to the field of pharmacogenetics, where we assess what type of medication and in what dosage should be delivered to a given person. All these profiles would be predetermined, and this information would be made available to the doctor or dentist, who would then use it to make decisions.

Audience: Dr. Joana, how does the Belgian government regulate a market-based dental model?

Joana Carvalho: In the Belgian case, the market-based approach works because it's a private model. There are a series of regulations in place and, therefore, the entire service is controlled. In addition, the government subsidizes the treatment, and health insurance companies exercise tight control over everything that is done. Everything is computerized and controlled. So it's part of an organization. Nevertheless, I believe the most important point here is the type of training the practitioners undergo, including in the university. I think that, for a long time here in Brazil, training was not focused on oral health. And now is the time to do this. We spend a lot of time preparing the specialized dentists. I think the market will also change depending on how we train our students. It will take some time.

Maria Fidela: In our country, the Pro-Saúde (Pro Health) Program is on the right track and we're starting to train professionals not only in the area of dentistry, but also in healthcare in general.

Nilce Tomita: Leaving aside the issue of treatment funding or payment, the practice of dentistry must be based on quality and effectiveness, regardless of where people are treated. And that's something that we must bear in mind in our daily practice. The knowledge produced by the Brazilian dentistry sector must be used to enhance health promotion and ethics.

Tiago Sousa: Canada has an interesting initiative concerning health funding. The Brazilian Minister of Health, Dr. Alexandre Padilha, said in an interview that he is trying to implement the Canadian model, based on indicators. What are these indicators? How many visits were made at home? In dentistry these indicators are still not very well defined, but could involve how many treatments were initiated, how many were completed, how many supervised brushings were conducted, etc. These are collective actions, actions that enable us to measure health. Instead of funding to deal with disease and illness, you would fund the incentives to promote health.

Audience: Thirty years ago, in the first ABOPREV discussions, genetics was used to explain why some people did not have caries and periodontal diseases. But, today, it is being used to explain why these diseases affect a small group of people. What motivated this change in perspective?

Alexandre Vieira: There are certain genetic variations that increase susceptibility to diseases, and there are others that decrease the susceptibility to different diseases. This is a model that I have observed, for example, in obesity and cardiovascular diseases. So I think about diseases and genetic susceptibility in terms of these two perspectives. I focus on variations that may protect you from having caries, for example, or from experiencing diseases, but at the same time, it is perhaps more difficult to identify the variation that increases your susceptibility.

Mário Taba: Just to add to that, I should say that periodontal diseases began to be better understood very recently. We worked with indexes and information surveys based on a scoring method for the diseases. When you group these scores into different clusters, into different age groups, over the years, older patients have higher average scores, and younger patients have lower average scores. So, we identified two problems: the first is the belief that gingivitis and periodontal disease are the same disease. The other problem was a lack of understanding about the non-linear progression of periodontal dis- 
eases. Taking into consideration the age factor over time and the average values, we would have a natural line indicating that the disease would become more severe over the years. If we were to merely interpret the curve, we would reach the conclusion that the genetic factor is not very relevant. Nevertheless, when we begin to identify sub-groups with different behaviors, it becomes clear that the genetic aspect should be considered, because it shows that the susceptibility factor must be incorporated into this equation.

Jaime Cury: In my opinion, the big difference between caries and periodontal diseases is the fact that periodontal diseases have a strong genetic component, which is the host's response. This does not apply to caries. Dental caries is basically the result of a physical-chemical phenomenon caused by the development of a lesion and the only important biological factor would be saliva acting as a modulator.

Audience: How can ABOPREV contribute to reducing inequality regarding access to health services?

Nilce Tomita: I think ABOPREV's key contribution would be to support the creation of effective strategies to reduce inequality. Therefore, ABOPREV's role, at first, would be to raise awareness about this issue in meetings such as this one. Given the degree of knowledge we currently have about state-of-the-art dental practices, we must ask ourselves how we can make this experience available to dental practitioners on a daily basis, including those in the public healthcare area. This is also a challenge for ABOPREV.

If we are able to include dental care in the broader network of health services and make it a part of people's everyday lives, over time, this will result in a reduction in the restorative dental treatments. Thus, I believe there are several areas in which ABOPREV can contribute.

Tiago Sousa: In terms of oral health disparities, ABOPREV has encouraged researchers, professors, and students to reflect on these issues. Literature shows that the statistics are in fact quite different between regions, due to socioeconomic factors.

In my opinion, ABOPREV must take a political stand and, we, as a professional association, must also put pressure on the Ministry of Health, so that the government may become aware of these disparities. Apart from that, we must also propose strategies to tackle this issue, instead of simply waiting for the Ministry of Health to implement policies that we will either criticize or endorse. We must be proactive partners in these actions.

I'll give you an example. The Epidemiological Survey on oral health carried out in 2003 provides interesting data based on the analysis of nearly 180,000 people in Brazil. One percent of the population was classified as indigenous. However, only when we compare the indigenous versus non-indigenous groups are we able to see that indigenous populations are seven times more likely to never have gone to a dentist before.

Therefore, we should think about how we are positioning ourselves as an entity, as an organization. That's what we need to improve. We cannot simply wait for the Ministry of Health to implement strategies or policies.

A year ago, during the XV ABOPREV Meeting, which was also held in Brasília, Gilberto Pucca addressed the plenary and asked what we should do about the healthcare for indigenous populations. I was very glad to see this matter addressed, because less than one year after he raised awareness to this issue, the Brasil Sorridente Program was updated and now we have a specific area of the program focused on the indigenous groups.

This was not a result of the "SB Brazil 2003" data itself. It was a result of a political movement created to mitigate these inequalities, to take action and to fight against injustice. In my opinion, this is the role ABOPREV should have.

From an academic perspective, we've struggled, we've developed, and we've expanded the boundaries of knowledge. On the other hand, from a political standpoint, we are still taking our first steps, but we have what it takes to move forward.

Lilian de Paula: The main purpose of this symposium was to discuss Oral Health Promotion, Expanding the Boundaries of Knowledge. We first had a discussion about the difficulties of access to health services, the social disparities, and the recently published study about oral health in Brazil. We also had 
an overview of the European health system, specifically the Belgian model. After this, we had a broad debate about oral health promotion, involving issues such as the genetic factors related to caries and periodontal diseases.

I would now like to pass the floor to Prof. Jaime Cury, so that he can make his final remarks.

Jaime Cury: It's hard to act as moderator, but I feel that my role here was to instigate the debate by asking some questions to the speakers, who did an excellent job in presenting their arguments and defending their points of view. I would also like to say that, in science, there is no ultimate truth. We surrender to the facts, so this discussion is relevant because it allows us to evolve beyond the knowledge we already have, and improve the quality of life of the population.

I'm very pleased with the outcome of this meeting, which is also an opportunity to celebrate ABOPREV's thirty years of existence. I believe we have achieved a great deal in terms of encouraging people and prompting them to champion a cause and to conduct research. As Alexandre pointed out in the beginning of his presentation, for several decades, we at ABOPREV have been very open and clear in our discussions, which have always been based on the best evidence possible. 